\title{
ARTIFICIAL INTELLIGENCE DESIGN OF NUCLEAR SYSTEMS EMPOWERED BY ADVANCED MANUFACTURING*
}

\author{
Vladimir Sobes, Briana Hiscox, Emilian Popov, Marco Delchini, Richard Archibald, Cory \\ Hauck, Paul Laiu, Ben Betzler, Kurt Terrani
}

Oak Ridge National Laboratory
Oak Ridge, TN 37831-6170, USA

sobesv@ornl.gov

\begin{abstract}
Implicit in any engineering design is an underlying optimization problem, although the exact objective function to be optimized is rarely stated explicitly. Nuclear systems optimization is as old as the discipline of nuclear engineering. Advanced manufacturing in the nuclear industry has opened the door for the re-examination of optimization in a way in which it was not possible before, namely, determining the optimal geometry for a given objective function. A trivial example is the sphere as the shape that minimizes the volume (or mass) of bare fissile material in a critical configuration. However, the problem becomes less trivial under even the simplest of multiphysics considerations. In this work, we develop the solution methodology for finding the minimum volume geometric configurations under the multiphysics constraints of $1,500 \mathrm{pcm}$ excess reactivity and maximum fuel temperature of $618^{\circ} \mathrm{C}$ under forced-flow cooling conditions. Constraining the solution geometry only to right cylinders, surprisingly yields two disjoint solution regions. Flat, wide (disk-like) cylinders and tall, narrow (rod-like) cylinders both satisfy the constraints and yield very similar minimal volumes. However, the ultimate pursuit of this work is truly arbitrary geometry.
\end{abstract}

KEYWORDS: nuclear systems design, optimization, arbitrary geometry, artificial intelligence.

\section{INTRODUCTION}

Advanced manufacturing applications in the nuclear industry have the potential to revolutionize nuclear design. While current engineering design objectives may be consistent with objectives from the past, such as those intended to minimize the fuel cost or to limit the maximum fuel temperature for a given power level, the possible geometries that can be considered as candidate designs have changed. Optimizations applied to current designs in nuclear engineering are limited by traditional geometric shapes that are easy to manufacture with traditional processes: slabs as fuel plates, cylinders as fuel rods, spheres as fuel pebbles, axis-aligned coolant channels, and more. Advanced manufacturing allows for an approach to the nuclear design problem with arbitrary geometries that can enable previously unrealized optimizations in performance and cost. This work explores the application of arbitrary geometry to the nuclear engineering design process.

* Notice: This manuscript has been authored by UT-Battelle LLC under contract DE-AC05-00OR22725 with the US Department of Energy (DOE). The US government retains and the publisher, by accepting the article for publication, acknowledges that the US government retains a nonexclusive, paid-up, irrevocable, worldwide license to publish or reproduce the published form of this manuscript, or allow others to do so, for US government purposes. DOE will provide public access to these results of federally sponsored research in accordance with the DOE Public Access Plan (http://energy.gov/downloads/doe-public-access-plan). 
If advanced manufacturing techniques become widely used in the nuclear industry, then a design approach for nuclear systems that is based on artificial intelligence (AI) will significantly enhance the field. While AI will not replace the human designer, the use of AI algorithms will shift the designer's focus away from conjecturing good geometries to defining the design criteria to find optimal designs. In the three demonstration designs presented herein, the AI algorithm produced two results that initially seem counterintuitive to a human designer, but on further inspection, they have been deemed optimal. The exploration of these designs demonstrated how AI uses the physics of the problem to find optimal designs.

This project used AI algorithms to explore how the freedom of geometry made possible with advanced manufacturing techniques changes the optimization of nuclear designs. In this initial effort, the goal was not to produce one optimal design for a given application, but rather to research and establish a framework for nuclear system design optimization that includes the types of arbitrary geometry enabled by advanced manufacturing.

The long-term vision for this project is to develop the initial framework into a very general nuclear engineering design tool. One of the powerful aspects of the framework is the modular nature of the relationship between the multiphysics calculations, the geometry representation, and the optimization software. Therefore, it will be relatively straightforward to incorporate more physics modules into the performance evaluation of one candidate design. While the current framework only considers the neutronics, heat transfer, and fluid dynamics associated with the candidate designs, other criteria, such as structural mechanics, thermophysical fuel performance, economics, and fuel cycle capabilities, can easily be added without requiring many changes in either the geometry representation module or the optimization module.

\subsection{Physics Problem Setup}

\section{MULTIPHYSICS SIMULATIONS}

Early in the project, the multiphysics definition of the optimization problem was established to mimic the requirements for the Transformational Challenge Reactor (TCR) core design at the time (the design has evolved since then), with a few modifications for simplification. While the multiphysics definition of the problem determines the final theoretical optimal design, the exact definition is not viewed as essential to establishing the AI optimization framework.

For the initial exploration of the framework and the challenge problems, the decision was made to optimize the geometry of a single piece of nuclear material, which was modeled as suspended in the middle of a cylindrical shroud with forced coolant flowing from the bottom to the top (bottom-up).

The material definitions for the fuel and the coolant were fixed to the values in Table I. All the fuel properties were taken to be temperature-independent for simplicity.

Table I. Material properties for the fuel and the coolant used in the challenge problems.

\begin{tabular}{ll|ll}
\multicolumn{2}{c|}{ Fuel: $\mathrm{UO}_{2}$} & \multicolumn{2}{c}{ Coolant: $\mathrm{He}$} \\
\hline Enrichment & $19.75 \%$ & Inlet pressure & $6 \mathrm{MPa}$ \\
Density & $10.8 \mathrm{~g} / \mathrm{cc}$ & Inlet flow velocity & $10 \mathrm{~m} / \mathrm{s}$ \\
Thermal conductivity & $4 \mathrm{~W} / \mathrm{mK}$ & Inlet temperature & $425^{\circ} \mathrm{C}$
\end{tabular}

The multiphysics constraints on the design were as follows:

1. neutron multiplication of $1.020 \pm 500 \mathrm{pcm}$,

2. maximum fuel temperature of $600^{\circ} \mathrm{C} \pm 3 \%$, and

3. design power of $10 \mathrm{~kW} \pm 3 \%$. 
In this work, only the steady-state problem is solved. While $2,000 \mathrm{pcm}$ above critical is a lot, it should be viewed more as an arbitrary design goal. In this case we justify it by deferring reactivity control to later engineering solutions or optimization using more detailed multiphysics modeling. For this project, the design power was significantly reduced from the TCR design because the study was limited to a single piece of fuel instead of a fuel array.

The design objective was to minimize the fuel volume (i.e., mass, as the density was fixed). Nuclear engineering intuition dictates that the goal of minimizing the fuel volume with a constant component power will push any near-optimal design to the minimum allowed neutron multiplication and to the maximum fuel temperature. Therefore, constraints 1 and 2 were reformulated as a multiplication factor above 1.015 and a maximum fuel temperature below $618^{\circ} \mathrm{C}$, taking into account the allowable tolerances.

It was verified that in the multiphysics regime used for optimization in this effort, the fuel density changes are negligible with respect to both reactivity and fuel temperature. Furthermore, the temperature feedback coefficient for criticality is within the uncertainty of $\pm 500 \mathrm{pcm}$ for temperatures of up to $925^{\circ} \mathrm{C}$. While we do not neglect to Doppler-broaden the cross sections to the average operating temperatures, we do neglect the effect of Doppler reactivity feedback. That is, we only solve the neutron transport problem once at mean temperatures. Similarly, all changes in thermophysical properties (e.g., thermal conductivity) due to irradiation are ignored due to the very short irradiation time assumed for TCR. Photon transport was noted for heating calculations as well as neutron and photon heating in the coolant, but it was neglected as appropriate for the flow characteristics.

With approximations taken into account, only a pseudo-coupled neutronic/thermal-hydraulic problem was solved. The component power level to was fixed to $10 \mathrm{~kW}$, regardless of the criticality or fuel geometry, the relative power distribution was passed to the thermal-hydraulics solver. This is considered pseudocoupling because it is only one-way. For this set of challenge problems, only the impact of the power distribution on the temperature distribution is considered, with no feedback to the neutronics solution from the thermal hydraulics. This is justified by the calculations of negligible temperature feedback in the operating regime.

\subsection{Current Approach for Multiphysics Simulations}

State-of-the-art modeling and simulation codes used in the nuclear research community were used to model the physics described in the previous section. A coupling methodology was developed between the neutron transport code Shift [1] and Star CCM+ [2, 3]. However, to enable rapid development and testing of the framework, a low-fidelity surrogate model was developed that was found to be accurate to within several percent of the high-fidelity model, which is not sufficient for true design analysis but is sufficient to demonstrate these approaches. The surrogate model is below, followed by the full physics model.

\subsubsection{Low-fidelity (surrogate) physics model}

The coupling of the neutron transport and the thermal hydraulics in the surrogate model was accomplished the same way as in the high-fidelity model: the relative power density was passed from the neutron transport solve to the thermal-hydraulics solve. The solution for both the neutron transport and the thermal hydraulics was calculated only on the fuel for the surrogate model.

The neutron transport surrogate model is a one-group neutron diffusion model with zero flux boundary conditions. The one-group constants were determined to match the infinite homogeneous medium multiplication factor of 1.51723 and a critical sphere radius of $35.78 \mathrm{~cm}$. Zero boundary conditions for non-re-entrant boundaries were taken for simplicity, and the coolant in between the re-entrant surfaces was modeled with zero absorption and a large diffusion coefficient. 
The thermal-hydraulics surrogate model was a heat-diffusion model with the fuel thermal conductivity from Table I. The total component power was normalized to $10 \mathrm{~kW}$, and fixed temperature boundary conditions of $425^{\circ} \mathrm{C}$ were assumed.

\subsubsection{High-fidelity (full) physics model}

The ORNL-developed Shift Monte Carlo radiation transport code [1] is used for the neutronics solve to leverage its ideal scaling characteristics. The Shift code then produces the multiplication factor and a core fission tally distribution. These tallies are converted into power densities and normalized so that the total core power is $10 \mathrm{~kW}$. The power density distribution is then read into a Java code, which builds and meshes the geometry and runs the thermal-hydraulics code Star CCM+ on each core. Star CCM+ returns the maximum temperature of each candidate design. Then the maximum temperature and multiplication factor $\left(\mathrm{k}_{\mathrm{eff}}\right)$ is fed back into the optimization module. The optimization module proposes a new set of candidate designs. This process is repeated until a converged design is achieved.

In view of the diverse geometry configurations of the optimized designs, an accurate prediction of temperature distribution in the core component is necessary. It is important that the thermal-hydraulic model is capable of computing a conjugate (solid-fluid) heat transfer in arbitrary geometrical shapes. To achieve this, a computational fluid dynamics (CFD) approach is taken. This method allows complex surfaces to be discretized with finite volume techniques, and it allows the interface between the solid structure and the coolant (gas in this case) to be properly defined. On the solid side, a thermal diffusion of heat with a volumetric heat source is computed to determine the temperature distribution. The heat generated by nuclear fission is deposited in the core element according to the volumetric power distribution supplied by the reactor physics calculation.

\subsection{Optimization Approaches}

\section{OPTIMIZATION}

The statistics community has expended considerable effort developing emulators that can approximate functional forms given uncertain data [4] [5]. Gaussian processes (GPs) are the preferred methods for approximating both the mean field and covariance processes from arbitrary pointwise values under the assumption that the values are independently identically distributed $\mathrm{N}\left(0, \sigma^{2}\right)$ random variables [6]. These GP approximation methods can be used with the optimization data to approximate the global optimization surface given a limited sampling of the full optimization surface. The team has developed a method that statistically determines the next set of samples to be simulated. This is likely to minimize a given loss function and should maintain any set of constraints given all the available simulations that have been run. This method grows in accuracy as more samples are simulated. For the case of optimization of the annulus challenge problem (Subsection 4.3), GP optimization was able to determine a near optimal solution in a couple of hundred samples in total.

The pattern search (also known as direct search) method [7] [8] was applied for solving this topological optimization problem. Pattern search is a class of derivative-free optimization methods that determines the search direction by probing objective function values at sample points in the optimization domain. To incorporate the multiphysics constraints, they are built into the objective function as penalty terms. In the optimization procedure, the GP approximation is used to estimate the criticality and maximum temperature at a given point in the optimization domain; this avoids the prohibitive computational cost of extensive multiphysics simulations.

The pattern search method used here consists of two stages: a global random search and a local refined search. The global search stage randomly samples search points that are further away from the current location to explore the optimization domain, whereas the local search procedure probes adjacent points to guarantee convergence to local minimums. Full multiphysics simulations are then run on the convergent 
points, and the GP approximation is updated with the simulation results. Then a new iteration of pattern search is started.

\subsubsection{Genetic algorithms}

Genetic algorithms are optimization techniques that borrow ideas from the natural world, including mutation, breeding (crossover), and survival of the fittest. Like in the natural world, in a genetic algorithm, unique individuals are compared according to a set of criteria and are combined to create new, unique individuals. These are then added to the population, and the best are again selected and combined. While the general outline of the algorithm is simple, the execution can be complex. This is due to having to explore potentially complex ideas such as how individuals should be judged, how individuals should be created and mutated, and how heuristics can be imposed to reduce unnecessary computation. This has been explored by starting with simple geometries of solid spheres and cylinders and solved for neutron multiplication using the relatively quick but least-accurate one-dimensional diffusion equations. By using simple, quick methods to evaluate the fitness of individuals, other important parameters related to the task were explored, such as mutation rates and varying crossover methods. These studies were built upon further by switching to a more accurate discrete ordinates method for solving for criticality and temperature and adding the annulus geometry type.

\subsection{Introduction to the Challenge Problems}

\section{OPTIMAL GEOMETRY RESULTS}

The challenge problems described in the following subsections were designed to be progressively more difficult to gradually challenge the team's ability to

1. represent increasingly more arbitrary geometry,

2. perform reliable multiphysics calculations, and

3. perform optimization over an increasing number of free parameters.

The other considerations in the design of the challenge problems were reasonable expectations of the existence of a global optimum (or a deep and stable local optimum) and the possibility of cross-validation between the challenge problems.

In the following subsections, the critical sphere radius for the material definitions is $0.358 \mathrm{~m}$, which corresponds to a volume of $0.192 \mathrm{~m}^{3}$ and a surface area of $1.6 \mathrm{~m}^{2}$. The infinite homogeneous multiplication factor is 1.51 , and the maximum allowable fuel temperature was $618^{\circ} \mathrm{C}$.

\subsection{Cylinder Core}

The first step was to restrict the geometry to a right cylinder. This geometry is far from arbitrary and is described by two free parameters: radius $(\mathrm{R})$ and height $(\mathrm{H})$. As for all the challenge problems, the goal was to minimize the fuel volume while maintaining a neutron multiplication factor above 1.015 and a maximum fuel temperature below $618^{\circ} \mathrm{C}$. The cylinder is positioned in a shroud with bottom-up coolant flow, as described above.

The goal of this challenge problem was to start with a simple problem, which allowed the team to

- exercise each of the multiphysics modeling modules,

- establish the neutronics/thermal-hydraulics coupling,

- compare the surrogate physics model with the full-physics model, and

- get started on the optimization module with only two free parameters.

The AI solution to the cylinder core also provided the first nonintuitive solution. By restricting the geometry to a right cylinder, one of three possible solutions was expected to be optimal: (1) a flat disk like a coin; (2) a compact cylinder with an $\mathrm{R} / \mathrm{H}$ ratio near 0.5 , like light water reactor fuel pellet; or (3) a long, thin cylinder like a fuel rod. The AI optimization found two near-equivalent solutions to the posed 
problem that were disjoint. The two solutions (within two significant figures) were combinations of $\mathrm{R}=$ $0.67 \mathrm{~m}, \mathrm{H}=0.37 \mathrm{~m}$ and $\mathrm{R}=0.27 \mathrm{~m}, \mathrm{H}=2.3 \mathrm{~m}$. These are the only two solutions, and no solution exists in between. Figure 1 gives a visualization of the two solutions.

Although it was not intuitively anticipated at first, the reasoning behind the two AI solutions was soon apparent. In this case, a multiphysics problem involving criticality and heat transfer is being solved. Criticality can be viewed, in a simplified manner, as the balance between fuel volume, which increases criticality, and neutron leakage (non-reentrant surface area), which decreases criticality. For heat transfer, the same two meta-parameters play a role, but in the opposing direction (as this study is trying to minimize the maximum fuel temperature). Fuel volume increases the maximum fuel temperature, and surface area decreases the maximum fuel temperature. Both of the solutions found by the AI algorithm had nearly the same volume of $0.52 \mathrm{~m}^{3}$ and the same surface area of $4.4 \mathrm{~m}^{2}$. On the other hand, in the subsequent challenge problems, no degenerate optimal solutions were found, even for geometries with equivalent surface-to-volume ratios as more complex neutronics, thermodynamic, and fluid flow considerations come into play.

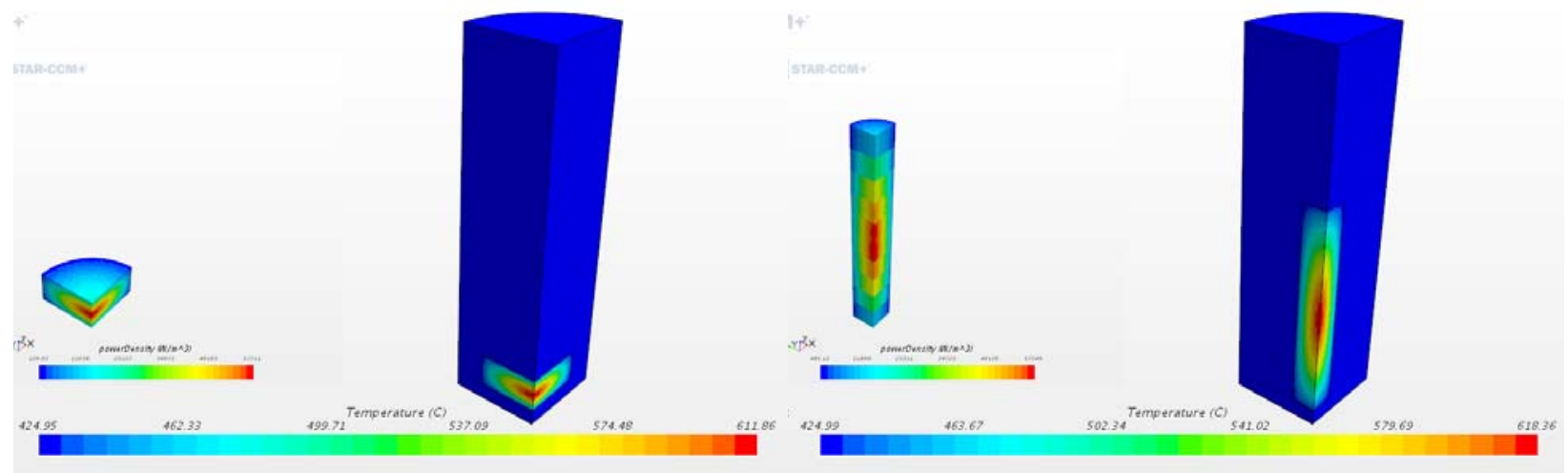

Figure 1. Visualization of two equivalent solutions for the cylinder core. Full geometry (quarter symmetry) is shown on left of each respective solution, and the CFD solution is on the right. The power density distribution is inserted in the CFD plot on the left. Left: $R=0.67 \mathrm{~m}, \mathrm{H}=0.37 \mathrm{~m}$. Right: $\mathrm{R}=\mathbf{0 . 2 7} \mathrm{m}, \mathrm{H}=\mathbf{2 . 3 \mathrm { m }}$

\subsection{Annulus Core}

The annulus core addressed the same problem formulation as before, but it allowed for the geometry to be parameterized by three free parameters: radius of the inner cylindrical flow channel, the outer radius of the fuel annulus, and the height of the fuel. The annulus core presented several new challenges:

- In both the surrogate model and the full multiphysics simulations, the reentrant geometry of the inner, cylindrical cooling channel had to be addressed.

- The optimization space increased to three degrees of freedom.

- The two radii parameters had to be restricted so that the inner radius was strictly smaller than the outer radius.

- This allowed for experimenting with the dramatic change in performance that results from introducing a hole in the design

The AI optimization found the optimal design to be an annulus with an inner cooling channel radius of $0.057 \mathrm{~m}$, an outer fuel radius of $0.34 \mathrm{~m}$, and a fuel height of $0.70 \mathrm{~m}$. In this case, the volume was $0.25 \mathrm{~m}^{3}$, a reduction of more than $50 \%$ from the cylinder core (due to reduced neutron leakage) and only $30 \%$ above the minimum volume to maintain criticality at any maximum fuel temperature. The surface area for this design was $2.5 \mathrm{~m}^{2}$. Figure 2 shows the relative proportions of the optimal annulus core. 


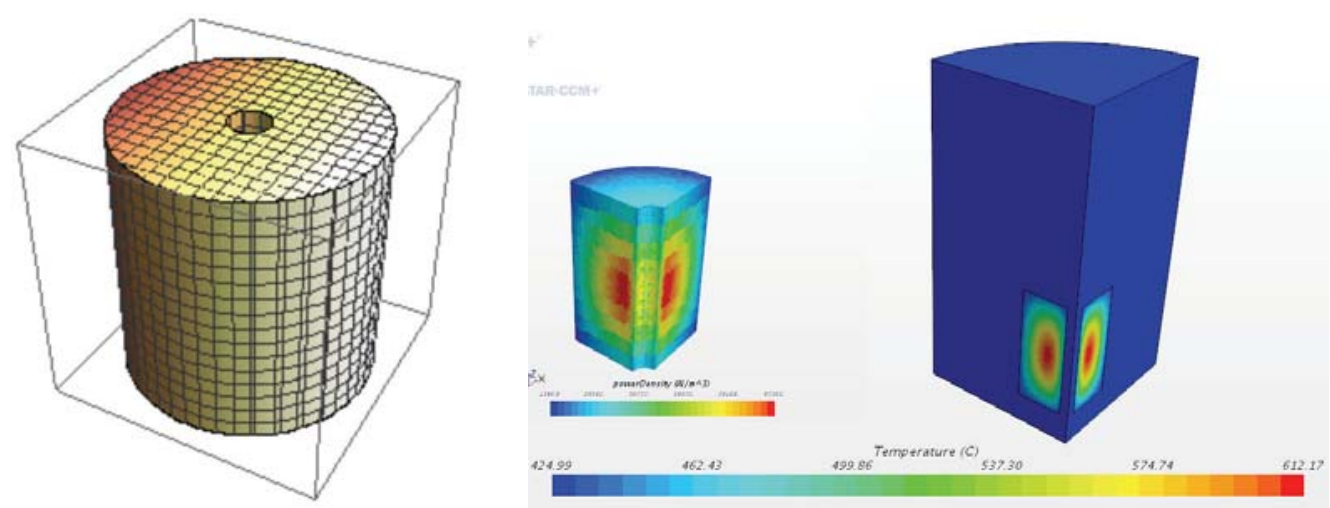

Figure 2. Relative proportions of the optimal annulus core: geometry (left), and CFD results (right).

\subsection{Cone Core}

The cone core is the first design in the series of challenge problems to diverge from traditionally manufacturable geometries and to seek out improved performance by considering simple shapes that could easily be made with advanced manufacturing but are difficult to manufacture using traditional methods. The cone core was a variant of the annulus core, and the inlet and outlet radii for both the inner cooling channel and the outer fuel radius could vary independently. The cone core presented a new set of challenges:

- The optimization space has increased to five free parameters: inlet inner radius, inlet outer radius, outlet inner radius, outlet outer radius, and height. The respective radii also had to be restricted.

- This was the first design to really challenge the fluid dynamics aspect of the calculations. As the cone core either became tapered into the flow or blunted, the fluid dynamics changed.

- As the surrogate model did not include a fluid dynamics approximation, it could not be used for the scoping studies.

Some possible candidate geometries are presented in Figure 3. Figure 3 visually presents only the effect on the outside of the geometry. The cone core is a variant of the annulus core where the outside of the fuel had the freedom to be tapered into the bottom-up coolant flow or blunted. The internal flow channel also had the freedom to either constrict the cooling channel or expand from the bottom to the top.

The AI optimization found that the optimal design had an inlet inner radius of $0.07 \mathrm{~m}$, an inlet outer radius of $0.37 \mathrm{~m}$, an outlet inner radius of $0.05 \mathrm{~m}$, an outlet outer radius of $0.35 \mathrm{~m}$, and a height of $0.64 \mathrm{~m}$.
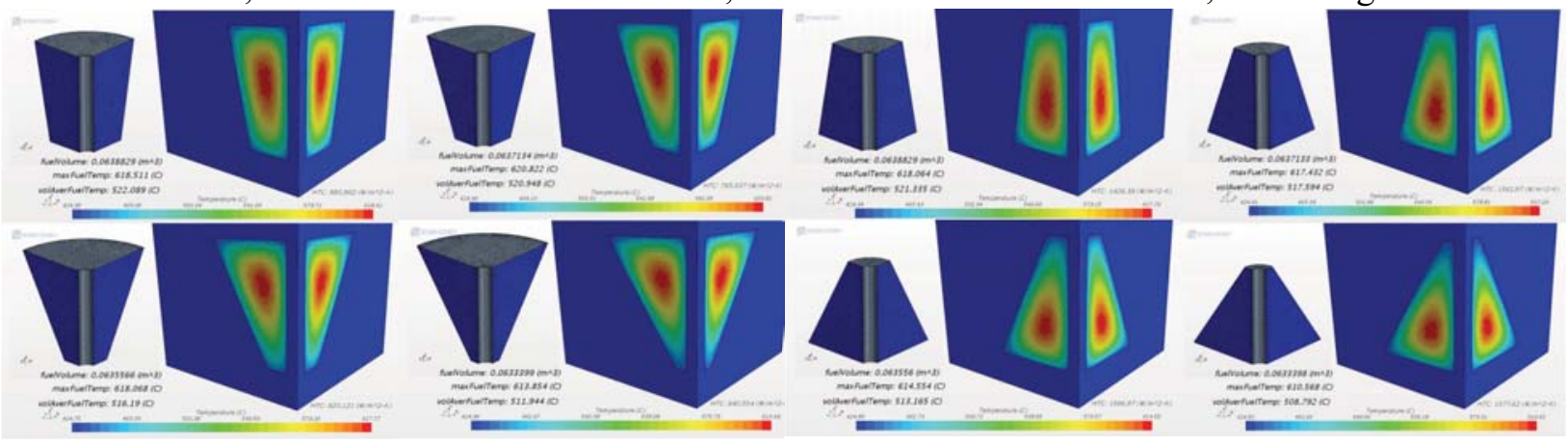

Figure 3. Candidate designs for the cone core. Showing the tapering of the outside of the geometry (left four figures) and the blunting of the outside of the geometry (right four figures).

The cone core was the second unintuitive result that the AI algorithm provided. As this was the first experience trying to optimize the flow around the fuel piece, an answer was expected that would taper the 
nose (inlet) of the cone core to smooth out the flow. This expectation was the opposite of what the AI algorithm found to be optimal.

The optimal cone-like core configuration is in the geometry shape of a truncated annular cone, with the inlet surface being larger than the outlet (blunt body). A more detailed analysis was performed to confirm the correctness of the optimization algorithm by analyzing the cooling efficiency of the optimal geometry.

\section{BIBLIOGRAPHY}

[1] T. M. Pandya, S. R. Johnson, T. M. Evans, G. G. Davidson, S. P. Hamilton and A. T. Godfrey, "Implementation, capabilities, and benchmarking of Shift, a massively parallel Monte Carlo radiation transport code," Journal of Computational Physics, vol. 308, pp. 239-272, 2016.

[2] S. J. Osher and R. P. Fedkiw, Level Set Methods and Dynamic Implicit Surfaces, Springer-Verlag, 2002.

[3] J. A. Sethian, Level Set Methods and Fast Marching Methods: Evolving Interfaces in Computational Geometry, Fluid Mechanics, Computer Vision, and Materials Science, Cambridge: University Press, 1999.

[4] D. Higdon, M. Kennedy, J. C. Cavendish, J. A. Cafeo and R. D. Ryne, "Combining field data and computer simulations for calibration and prediction," SIAM Journal of Scientific Computing, vol. 26, pp. 448-446, 2004.

[5] M. C. Kenned and A. O'Hagan, "Bayesian calibration of computer models (with Discussion)," Journal of the Royal Statistical Society - Series B, vol. 63, pp. 425-464, 2001.

[6] C. E. Rasmussen and C. K. Williams, "Gaussian Processes for Machine Learning," Adaptive Computation and Machine Learning, 2005.

[7] T. G. Kolda, R. M. Lewis and V. Torczon, "Optimization by direct search: New perspectives on some classical and modern methods," SIAM reivew, vol. 45, no. 3, pp. 385-482, 2008.

[8] C. Audet and J. E. Dennis, "Analysis of generalized pattern searches," SIAM Journal on optimizaiton, vol. 13, no. 3, pp. 889-903, 2002.

[9] L. Eca and M. Hoekstra, "A procedure for the estiamtion of the numerical uncertainty of CFD calculations based on grid refinement studies," Journal of Computational Physics, pp. 104-130, 2014.

[10] S. Osher and J. A. Sethian, "Fronts propagating with curvature-dependent speed: Algorithms based on Hamilton-Jacobi formulations," Journal of Compuational Physics, pp. 12-49, 1988.

[11] Siemens, "STAR-CCM+ documentaion and code description," [Online]. Available: https://www.plm.automation.siemens.com/global/en/products/simcenter/STAR-CCM.html.

[12] M. B. Giles, "Multilevel Monte Carlo path simulation," Operations Research, vol. 56, no. 3, pp. 607-617, 2008.

[13] B. Peherstorfer, K. Willcox and M. Gunzberger, "Survey of multifidelity methods in uncertainty propagation, inference, and optimization," Siam Review, vol. 60, no. 3, pp. 550-591, 2018.

[14] V. Torczon, "On the convergence of pattern search algorithms," SIAM Journal on optimization, vol. 7, no. 1, pp. 1-25, 1997.

[15] J. Gero, "Design Optimization," Academic Press, 1985.

[16] O. Sigmund and K. Maute, "Topology optimization approaches, A compartive review," Structural Multidisciplinary Optimization, vol. 48, pp. 1031-1055, 2013. 